\title{
Europium as a lodestar: diagnosis of radiogenic heat production in terrestrial exoplanets
}

\section{Spectroscopic determination of Eu abundances in $\alpha$ Centauri AB ${ }^{\star}$}

\author{
H. S. Wang ${ }^{1}$, T. Morel², S. P. Quanz ${ }^{1}$, and S. J. Mojzsis ${ }^{3,4}$ \\ ${ }^{1}$ Institute for Particle Physics and Astrophysics, ETH Zürich, Wolfgang-Pauli-Strasse 27, 8093 Zürich, Switzerland \\ e-mail: haiwang@phys .ethz.ch \\ ${ }^{2}$ Space sciences, Technologies and Astrophysics Research (STAR) Institute, Université de Liège, Quartier Agora, \\ Allée du 6 Août 19c, Bât. B5C, 4000 Liège, Belgium \\ e-mail: tmorel@uliege.be \\ ${ }^{3}$ Department of Geological Sciences, University of Colorado, UCB 399, 2200 Colorado Avenue, Boulder, CO 80309-0399, USA \\ e-mail: stephen.mojzsis@colorado.edu \\ ${ }^{4}$ Institute for Geological and Geochemical Research, Research Centre for Astronomy and Earth Sciences, \\ Hungarian Academy of Sciences, 1112 Budapest, Hungary
}

Received 9 May 2020 / Accepted 11 October 2020

\begin{abstract}
Context. Long-lived radioactive nuclides, such as ${ }^{40} \mathrm{~K},{ }^{232} \mathrm{Th},{ }^{235} \mathrm{U}$, and ${ }^{238} \mathrm{U}$, contribute to persistent heat production in the mantle of terrestrial-type planets. As refractory elements, the concentrations of $\mathrm{Th}$ and $\mathrm{U}$ in a terrestrial exoplanet are implicitly reflected in the photospheric abundances of the stellar host. However, a robust determination of these stellar abundances is difficult in practice owing to the general paucity and weakness of the relevant spectral features.

Aims. We draw attention to the refractory, $r$-process element europium, which may be used as a convenient and practical proxy for the population analysis of radiogenic heating in exoplanetary systems.

Methods. As a case study, we present a determination of Eu abundances in the photospheres of $\alpha$ Cen A and $\mathrm{B}$ with high-resolution HARPS spectra and a strict line-by-line differential analysis. To first order, the measured Eu abundances can be converted into the abundances of ${ }^{232} \mathrm{Th},{ }^{235} \mathrm{U}$, and ${ }^{238} \mathrm{U}$ with observational constraints, while the abundance of ${ }^{40} \mathrm{~K}$ is approximated independently with a Galactic chemical evolution model.

Results. Our determination shows that europium is depleted with respect to iron by $\sim 0.1$ dex and to silicon by $\sim 0.15$ dex compared to solar in the two binary components. The loci of $\alpha \mathrm{Cen} \mathrm{AB}$ at the low-ends of both [Eu/Fe] and [Eu/Si] distributions of a large sample of FGK stars further suggest significantly lower potential of radiogenic heat production in any putative terrestrial-like planet (i.e. $\alpha$-Cen-Earth) in this system compared to that in rocky planets (including our own Earth) that formed around the majority of these Sun-like stars. Based on our calculations of the radionuclide concentrations in the mantle and assuming the mantle mass to be the same as that of our Earth, we find that the radiogenic heat budget in an $\alpha$-Cen-Earth is $73.4_{-6.9}^{+8.3} \mathrm{TW}$ upon its formation and $8.8_{-1.3}^{+1.7}$ TW at the present day, which is $23 \pm 5 \%$ and $54 \pm 5 \%$ lower than that in the Hadean Earth $(94.9 \pm 5.5 \mathrm{TW})$ and in the modern Earth $(19.0 \pm 1.1 \mathrm{TW})$, respectively.

Conclusions. As a consequence, mantle convection in an $\alpha$-Cen-Earth is expected to be overall weaker than that of Earth (assuming other conditions are the same), and thus such a planet would be less geologically active, suppressing its long-term potential to recycle its crust and volatiles. With Eu abundances being available for a large sample of Sun-like stars, the proposed approach can extend our ability to predict the nature of other rocky worlds that can be tested by future observations.
\end{abstract}

Key words. stars: abundances - stars: individual: $\alpha$ Cen A - stars: individual: $\alpha$ Cen B - planets and satellites: composition

\section{Introduction}

A major goal of modern astronomy is to better define the nature of exoplanets and understand planet formation and evolution, as well as life prospects, in a cosmic perspective. To this end, communities in Earth sciences, exoplanet science, and stellar astrophysics have been increasingly joining forces through existing and future ground- and space-based astronomical infrastructures (e.g. Gaia, VLT, Kepler, TESS, ELT, JWST, PLATO) and/or collaboration networks (e.g. NExSS, PlanetS, GALAH).

Through spectroscopic observations of their photospheres, we can decipher the elemental compositions of stars, which

\footnotetext{
* Based on observations collected at the La Silla Observatory, ESO (Chile) with the HARPS spectrograph.
}

in turn yield unique insights into the formation and bulk compositions of the planets formed around them (Bond et al. 2010; Pagano 2014; Wang et al. 2019a; Doyle et al. 2019; Liu et al. 2020). The resulting data can be used to infer gross geodynamical properties (including interior, surface, and atmosphere) of terrestrial-like exoplanets (Frank et al. 2014; Noack et al. 2017; Hinkel \& Unterborn 2018; Wang et al. 2019b; Shahar et al. 2019).

Radiogenic heat generated by the decay of the long-lived radionuclides $\left({ }^{40} \mathrm{~K},{ }^{232} \mathrm{Th},{ }^{235} \mathrm{U}\right.$, and $\left.{ }^{238} \mathrm{U}\right)$ contributes a timedependent but significant proportion of the Earth's internal heat (e.g. Gando et al. 2011; Lenardic et al. 2011; Frank et al. 2014; Nimmo 2015), which powers mantle convection that leads to persistent plate tectonics, plume activity, and other forms of volcanism that eventually made our planet habitable (Sleep 2007; 
Lugaro et al. 2018; Lingam \& Loeb 2020; Seales \& Lenardic 2020). Knowing the abundances of these long-lived, heatproducing isotopes in other rocky planets is therefore critical to the assessment (to first order) of the geological activity of these planets. It is noteworthy that the heat-producing shortlived nuclides ${ }^{26} \mathrm{Al}$ and ${ }^{60} \mathrm{Fe}$ are important heating sources in shaping the composition of planetesimals in the early Solar System (Lichtenberg et al. 2016) but become effectively extinct after $\sim 3$ Myr and essentially do not contribute to heat production in already formed planets (Frank et al. 2014).

The other main source of planetary internal heat is the dynamical or gravitational energy, inherited from planet formation and core-mantle segregation; this declines with time owing to secular cooling (Stevenson 2003; Lyubetskaya \& Korenaga 2007). Radiogenic heating also declines with time but in different ways: it follows the exponent decay of radionuclides. An expression of the relative ratio of radiogenic heating and gravitational energy is dubbed the Urey ratio/number, which is defined as the ratio of the instantaneous radiogenic heat production to the total surface heat flow of the planet at that time; proposed values range from approximately 0.3 to 0.9 for the case of the Earth (Schubert et al. 2001). Placing direct constraints on the Urey number for exoplanets is not possible, however, because information related to the multitude of possible disc environments, planet formation histories, and evolution scenarios is scarce. With these important caveats, we can start with the host stellar abundances to make preliminary inferences about rocky worlds around other stars (Santos et al. 2017; Doyle et al. 2019; Wang et al. 2019b; Liu et al. 2020), which can be verified (or refuted) as new observational techniques are developed.

In addition, challenges also exist in spectroscopically determining the abundances of the principal long-lived radioactive elements in planet-hosting stars (e.g. Unterborn et al. 2015; del Peloso et al. 2005; Botelho et al. 2019). For instance, the Th abundance is commonly determined through modelling of a single line (Th II 24019.1) that is heavily blended with stronger features of other elements (e.g. Fe and $\mathrm{Ni}$ ) and very sensitive to continuum placement. As a result, selecting other elements that can act as proxies and are easier to measure is a convenient and practical approach, especially for a population analysis of radiogenic heating of potential rocky worlds around other stars.

Because $U$ and Th are pure neutron-capture (rapid-) $r$-process elements (Simmerer et al. 2004; Bisterzo et al. 2014), the surrogates should also be produced through this nucleosynthesis channel as much as possible. Based on this criterion, Ir and Eu, which contribute 98.4 and $94.0 \%$ by $r$-process, respectively (Bisterzo et al. 2014), are the most suitable proxy candidates for $\mathrm{U}$ and $\mathrm{Th}$. Because the Eu abundances are considerably easier to determine in extrasolar systems, we regard it as the appropriate choice here (as supported by Yong et al. 2008). Other proxies for long-lived radionuclides are also discussed in the literature, for instance, $\mathrm{Hf}, \mathrm{Bi}$, and $\mathrm{Tl}$ (Sneden et al. 2008; Wilford 2011). However, Eu must clearly be preferred given the large sets of Eu abundances for FGK dwarfs (e.g. Pagano 2014; Delgado Mena et al. 2017; Battistini \& Bensby 2016; Mishenina et al. 2016; Guiglion et al. 2018), which overwhelmingly exceed what is available for other proxy candidates (see e.g. Hinkel et al. 2019). The abundance measurements of the latter proxies are either restricted to stars with very peculiar chemical patterns (e.g. Roederer et al. 2018) or are not possible at all, as illustrated by the absence of their lines in the solar photospheric spectrum (e.g. Bi and Tl; Grevesse et al. 2015). In addition, because the $r$-process contribution to Eu is high, it has been used extensively together with a typical $s$-process element (e.g. Ba) as a chemical clock in nucleosynthesis as well as to assess the $r$-process enrichment in galaxy chemical evolution histories (Mashonkina \& Gehren 2000; Jacobson \& Friel 2013; Ji et al. 2016; Bisterzo et al. 2016; Skúladóttir et al. 2019).

Observations of both $\mathrm{Eu}$ and $\mathrm{Th}$ abundances in solar analogues with a wide range of ages $(0-10 \mathrm{Ga})$ have shown that the $[\mathrm{Eu} / \mathrm{H}]$ and initial $[\mathrm{Th} / \mathrm{H}]$ abundance ratios evolve in lockstep: [Th/Eu] is solar within 0.04 dex during the Galactic thin-disc evolution (Botelho et al. 2019). Measurements in meteoritic and in Galatic halo stars have also shown a nearly constant U/Th production ratio $\left(0.571_{-0.031}^{+0.037}\right.$; Dauphas 2005). In addition, Eu is a refractory element (whose condensation temperature is even slightly higher than that of $\mathrm{Fe}$ and $\mathrm{Ni}$; see Lodders 2003 and Wood et al. 2019), implying that its abundance in a terrestrial planet is representative and closely related to that of the stellar host. Finally, Eu has stable isotopes, and an age correction is thus not necessary to estimate its pristine abundance at the time of planet formation. However, we must note in advance that ${ }^{40} \mathrm{~K}$ cannot be directly/indirectly inferred from Eu because its nucleosynthesis pathways are distinct (Clayton 2003; Zhang et al. 2006) and its nature is volatile (Wang et al. 2019a). Instead, alternative assumptions (detailed below) have to be made regarding ${ }^{40} \mathrm{~K}$.

The recognition that $\mathrm{Eu}$ is a suitable reference element for long-lived radionuclides (excluding ${ }^{40} \mathrm{~K}$ ) is by no means new (Pagel 1989), but it can be argued that its usefulness as a proxy for (partial) internal heat production has not be been fully recognised by the exoplanet community (e.g. Kite et al. 2009). The vast majority of stars potentially hosting terrestrial-like planets do not have Eu abundances available (e.g. Schuler et al. 2015). As demonstrated in the following, Eu abundances can be spectroscopically determined with relative ease even in stars that are not necessarily (very) bright, because the strength of the Eu II lines in the blue spectral range is moderate. We therefore also hope that our study will motivate more widespread abundance determinations of this element in stars with potentially rocky planets.

As a case study, we turn our attention to our nearest Sunlike star systems, $\alpha$ Cen AB, at only $\sim 1.3$ pc. Although no planet orbiting either binary component has been confirmed yet (e.g. Dumusque et al. 2012; Hatzes 2013; Rajpaul et al. 2016), various numerical simulations support the contention that the stability of planetary orbits can persist within the so-called habitable zones of these two binary stars (Andrade-Ines \& Michtchenko 2014; Quarles \& Lissauer 2016, 2018). If a small (potentially rocky) planet is discovered in the habitable zone of either $\alpha$ Cen A or B, the intensive discussion about the nature of such a planet would be brought to the forefront. The detailed chemical composition of $\alpha$ Cen AB recently revisited by Morel (2018, hereafter M18) enables such a discussion to be made quantitatively, except for the planetary internal heat budget, partially owing to the lack of abundances of heat-producing elements (U, $\mathrm{Th}$, and K) or their proxies. In the context of exoplanet studies, and in particular, our modelling of the radiogenic heat power in the putative planets orbiting $\alpha$ Cen $\mathrm{A}$ or $\mathrm{B}$, we present in this work our spectroscopic analysis and results of $\mathrm{Eu}$ abundances of both stars (Sects. 2 and 3), followed by a comparison with previous Eu analyses for $\alpha$ Cen A/B (Sect. 4.1) and with Eu abundances of other stars (Sect. 4.2). We provide an assessment of the radiogenic heat budget for potential terrestrial planets in the system (Sect. 4.3), together with an analysis of the limitations to our approach (Sect. 4.4). Our conclusions are summarised in Sect. 5. 


\section{Analysis}

Aiming for a homogeneous spectroscopic analysis of the $\mathrm{Eu}$ abundances in $\alpha$ Cen $\mathrm{AB}$, we closely followed the procedures employed in M18. However, two aspects were necessarily different. First, the diagnostic Eu lines are either located in the far blue or are otherwise weak. This means that the HARPS spectra used by M18, which do not extend below $4800 \AA$ and do not have an extremely high signal-to-noise ratio $(\mathrm{S} / \mathrm{N})$, are not suitable. M18 were unable to measure the Eu II $\lambda 6645$ line confidently with an equivalent width (EW) below $10 \mathrm{~m} \AA$ in $\alpha$ Cen $\mathrm{AB}$. Second, the Eu II lines are in general strongly blended and broadened by isotopic and hyperfine (HFS) splitting, which calls for an abundance determination relying on spectral synthesis instead of an analysis based on EWs.

For the purpose of this work, our analysis is based on highresolution HARPS spectra retrieved from the European Southern Observatory (ESO) archives. These have a resolving power, $R$, of about 115000 and cover the spectral range 3780-6910 A. For the solar spectrum to be used as reference, we averaged with a weight that depends on the mean $\mathrm{S} / \mathrm{N}$ of all the available exposures of asteroids with an $\mathrm{S} / \mathrm{N}$ above 100. Observations of a point-like source are preferred (e.g. Gray et al. 2000), while coadding spectra from various reflecting bodies is not an issue (e.g. Bedell et al. 2014). For $\alpha$ Cen AB, we collected all spectra with $375<S / N<400$. Spectra with a higher $\mathrm{S} / \mathrm{N}$ were ignored to avoid saturation problems. For $\alpha$ Cen A, we only considered the numerous spectra obtained over five consecutive nights by Bazot et al. (2007) for their asteroseismic analysis. After rejecting the spectra with Eu features affected by cosmic rays or telluric features (i.e. the molecular lines of the Earth's atmosphere), we had a total of 177, 283, and 284 spectra for the Sun, $\alpha$ Cen A, and $\alpha$ Cen B, respectively. However, about $30 \%$ of the exposures were discarded for the analysis of Eu II $\lambda 6645$ in $\alpha$ Cen B because of fringing patterns in the red. All spectra were corrected for radial-velocity shifts prior to co-adding based on the precise cross-correlation (CCF) data from the instrument reduction pipeline. The mean spectra were normalised to the continuum by fitting low-order Legendre polynomials using standard tasks implemented in the IRAF ${ }^{1}$ software. To ensure the highest consistency, this procedure was identical for all three stars.

Our results are based on a line-by-line differential analysis relative to the Sun. We make use of plane-parallel, 1D MARCS model atmospheres (Gustafsson et al. 2008) and the 2017 version of the line-analysis software MOOG originally developed by Sneden (1973). M18 carried out the analysis using various line lists taken from the literature. However, only two include Eu features: Meléndez et al. (2014, hereafter Me14) considered Eu II $\lambda 3819.7,3907.1,4129.7$, and 6645.1, while the study of Reddy et al. (2003, hereafter Re03) only included Eu II $\lambda 6645.1$. We did not consider Eu II $\lambda 3819.7$ any further because this weak line is heavily blended and difficult to model properly, as discussed by Lawler et al. (2001). The HFS data are taken from Ivans et al. (2006) and assume the ${ }^{151} \mathrm{Eu} /{ }^{153} \mathrm{Eu}$ isotopic ratio from Chang et al. (1994). The study of Ivans et al. (2006) is an improvement over the reference work of Lawler et al. (2001), as it provides updated Eu transition data. About 30 HFS components were taken into account for each line we studied. The

\footnotetext{
1 IRAF is distributed by the National Optical Astronomy Observatories, operated by the Association of Universities for Research in Astronomy, Inc., under cooperative agreement with the National Science Foundation.
}

lines of other elements in the relevant spectral ranges were modelled using data retrieved from the VALD3 atomic database ${ }^{2}$ and assuming the abundances of M18. For the minor species not included in M18, we scaled the abundances according to $[\mathrm{Fe} / \mathrm{H}]$ because estimates in the literature are either not robust or for the most part simply not available. We note that this assumption has no effect on our main results. The dissociation energies implemented in MOOG were assumed for the molecular species. To fit the solar spectrum, we adopted projected rotational and macroturbulent velocities of 1.8 and $3.1 \mathrm{~km} \mathrm{~s}^{-1}$, respectively. For $\alpha$ Cen AB, we assumed the values quoted by Bruntt et al. (2010). Instrumental broadening was also taken into account: the value was first adjusted based on a fit of the relatively unblended Fe II 4128.7 line (see e.g. Koch \& Edvardsson 2002), and then scaled as a function of wavelength according to the full width at half maximum (FWHM) of lines measured in calibration lamps.

Our strategy was first to adjust the oscillator strengths of lines in vicinity of the Eu feature of interest to optimise the quality of the fit in the Sun. The solar mixture we adopted (Grevesse et al. 2007) is consistent with that adopted for the computation of the MARCS model atmospheres. For the solar parameters, we adopted an effective temperature, $T_{\text {eff }}$, of $5777 \mathrm{~K}$, a surface gravity, $\log g$, of 4.44 , and a microturbulence, $\xi$, of $1 \mathrm{~km} \mathrm{~s}^{-1}$. Finally, to improve the fit in $\alpha$ Cen $\mathrm{AB}$, we altered the abundances of elements whose lines significantly affect the Eu feature within their uncertainties. Similarly to a number of previous studies (e.g. del Peloso et al. 2005; Lawler et al. 2001; Peek 2009), it was occasionally necessary to include some artificial Fe I lines in the line list because of unaccounted-for absorption. The changes to the initial VALD3 line list are summarised in Table 1. We stress that these slight adjustments lead to a noticeably better fit, but have little effect on the resulting Eu abundances. We modelled $\alpha$ Cen AB by adopting the stellar parameters $\left(T_{\text {eff }}, \log g\right.$ and $\xi$ ) derived by M18 for the relevant line list. As for the other elements studied in M18, we used the "unconstrained" results quoted in his Table B.2 that are obtained without freezing the surface gravity to the asteroseismic value quoted by Heiter et al. (2015). Illustrative examples of the fits are shown in Fig. 1.

We determine for the Sun an average $\mathrm{Eu}$ abundance, $\left\langle\log \epsilon_{\odot}(\mathrm{Eu})\right\rangle=+0.46 \pm 0.05$, based on 1D model atmospheres and assuming local thermodynamic equilibrium (LTE). Theoretical calculations by Mashonkina \& Gehren (2000) indicate that the abundances determined from Eu II features must be corrected upwards in solar-like stars to account for departures from LTE. The non-LTE corrections for the Sun amount on average to +0.03 dex for Eu II $\lambda 4429$ and Eu II $\lambda 6645$. On the other hand, 1D-3D corrections appear to be negligible according to $\mathrm{CO}^{5} \mathrm{BOLD}$ hydrodynamical simulations (Mucciarelli et al. 2008). We therefore obtain a corrected solar abundance, $\left\langle<\log \epsilon_{\odot}(\mathrm{Eu})\right\rangle=+0.49 \pm 0.05$, that is fully compatible with the recommended meteoritic and photospheric values that lie in the range 0.51-0.52 (Grevesse et al. 2015, and references therein). However, given the differential nature of our analysis with respect to the Sun, we emphasise that the exact values of the absolute solar abundances have no bearing on our conclusions.

\section{Results}

The abundance analysis results are summarised in Table 2. Following M18, the random uncertainties were computed by adding in quadrature the line-to-line scatter, $\sigma_{\text {int }}$, and the uncertainties

2 http://vald.astro.uu.se/ 
Table 1. Changes made to the initial VALD3 line list.

\begin{tabular}{lllccl}
\hline \hline \multirow{2}{*}{ Ion } & \multirow{2}{*}{$\lambda(\AA)$} & \multirow{2}{*}{ LEP $(\mathrm{eV})$} & \multicolumn{2}{c}{$\log g f$} & \multirow{2}{*}{ Remark } \\
\cline { 4 - 5 } & & & This study & VALD3 & \\
\hline Eu II $\lambda 3907.1$ & & & & & \\
Fe I & 3906.9617 & 3.283 & -2.27 & -1.481 & $\log g f$ adjusted \\
Fe I & 3907.22 & $3.5^{(a)}$ & -2.63 & $\ldots$ & $\operatorname{artificial~line~}$ \\
Ce II & 3907.2876 & 1.107 & +0.24 & +0.320 & $\log g f$ adjusted \\
\hline Eu II $\lambda 4129.7$ & & & & & \\
Fe I & 4129.29 & $3.5^{(a)}$ & -2.95 & $\ldots$ & artificial line \\
Fe I & 4129.35 & $3.5^{(a)}$ & -3.1 & $\ldots$ & $\operatorname{artificial~line~}$ \\
Fe I & 4129.4600 & 3.397 & -1.89 & -1.970 & $\log g f$ adjusted \\
Fe I & 4129.53 & $3.5^{(a)}$ & -3.1 & $\ldots$ & $\operatorname{artificial~line~}$ \\
${ }^{12} \mathrm{CN}$ & 4129.6006 & 0.976 & -0.8 & -0.585 & $\log g f$ adjusted \\
Ti I & 4129.6429 & 2.239 & -1.8 & -1.424 & $\log g f$ adjusted \\
Fe I & 4129.956 & $3.5^{(a)}$ & -2.38 & $\ldots$ & $\operatorname{artificial~line~}$ \\
Fe I & 4130.0366 & 1.557 & -3.54 & -4.034 & $\log g f$ adjusted \\
Cr I & 4130.0614 & 2.913 & -2.6 & -0.840 & $\log g f$ adjusted \\
\hline Eu II $\lambda 6645.1$ & & & & & \\
${ }^{12} \mathrm{CN}$ & 6644.9153 & 1.066 & -1.825 & -2.008 & $\log g f$ adjusted \\
Si I & 6645.2099 & 6.083 & -2.4 & -3.156 & $\log g f$ adjusted \\
Fe I & 6645.3645 & 4.386 & -2.95 & -3.622 & $\log g f$ adjusted \\
${ }^{12} \mathrm{CN}$ & 6645.4038 & 0.956 & -2.087 & -1.887 & $\log g f$ adjusted \\
\hline
\end{tabular}

Notes. LEP is the lower excitation potential. ${ }^{(a)}$ Arbitrary value adopted following Lawler et al. (2001).

Eu II $\lambda 3907.11$

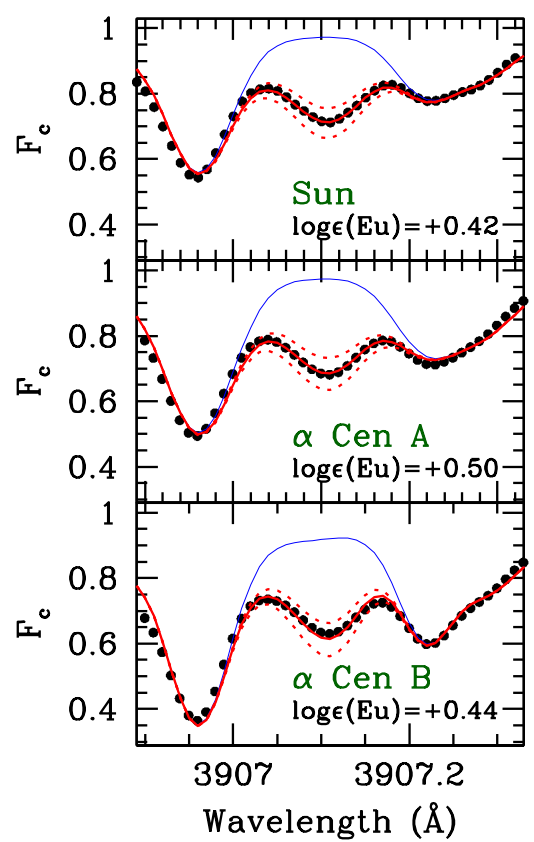

Eu II $\lambda 4129.72$

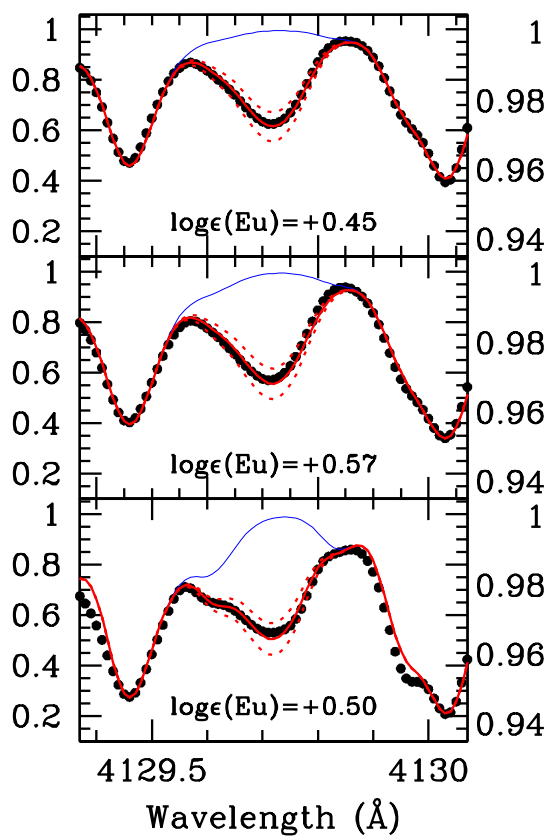

Eu II $\lambda 6645.10$

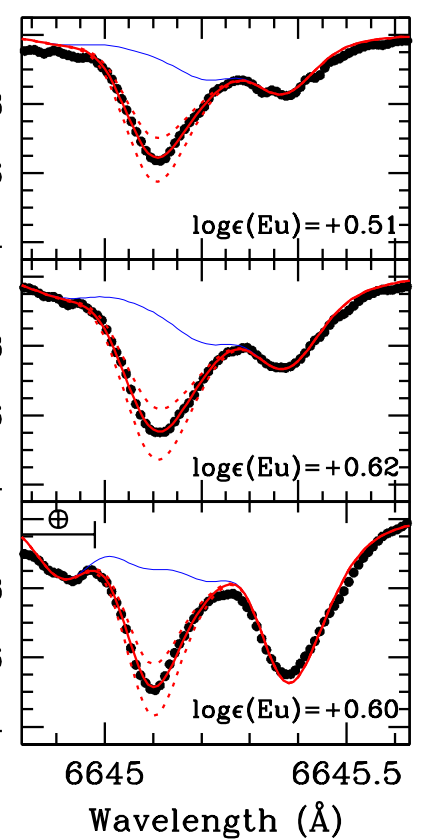

Fig. 1. Examples of fits to the Eu II spectral features in the Sun (top panels) and $\alpha$ Cen $\mathrm{AB}$ (middle and bottom panels) based on the line list of Me14. The solid red line shows the best-fitting synthetic profile, while the two dotted lines show the profiles for an Eu abundance deviating by $\pm 0.1 \mathrm{dex}$. The blue line shows the profile without Eu. The best-fitting abundance is indicated in each case. The ordinate scale for Eu II $\lambda 6645$ differs strongly. The Earth symbol in the bottom right panel marks the location of telluric features (the Sun and $\alpha$ Cen A are not significantly affected).

arising from errors in the stellar parameters. The larger uncertainties for $\alpha$ Cen B arise from the difficulty in modelling the spectrum of relatively cool stars exhibiting molecular features and the fact that the stellar parameters are much more sensitive to the choice of the iron line list (see M18).
Tests using Kurucz models ${ }^{3}$ indicate that the choice of the family of 1D model atmospheres affects $\alpha$ Cen $\mathrm{AB}$ and the Sun in a similar way and therefore cancels out to first order

3 http://kurucz.harvard .edu/grids.html 
Table 2. Abundance results for $\alpha \mathrm{Cen} \mathrm{AB}$ and comparison to values in the literature.

\begin{tabular}{lcccccc}
\hline \hline & \multicolumn{7}{c}{$[\mathrm{Eu} / \mathrm{H}]$} \\
\cline { 2 - 7 } & $\alpha$ Cen A & $\alpha$ Cen B & A-B & $\alpha$ Cen A & $\alpha$ Cen B & A-B \\
\hline This study & & & & & \\
Me14 line list & $+0.10 \pm 0.04(3)$ & $+0.05 \pm 0.06(3)^{(a)}$ & $+0.05 \pm 0.07(3)^{(a)}$ & $-0.12 \pm 0.05(3)$ & $-0.17 \pm 0.07(3)^{(a)}$ & $+0.05 \pm 0.08(3)^{(a)}$ \\
Re03 line list & $+0.15 \pm 0.06(1)$ & $+0.16 \pm 0.08(1)^{(a)}$ & $-0.01 \pm 0.10(1)^{(a)}$ & $-0.08 \pm 0.07(1)$ & $-0.07 \pm 0.09(1)^{(a)}$ & $-0.01 \pm 0.11(1)^{(a)}$ \\
Weighted average & $+0.12 \pm 0.04(3)$ & $+0.09 \pm 0.05(3)^{(a)}$ & $+0.03 \pm 0.06(3)^{(a)}$ & $-0.11 \pm 0.04(3)$ & $-0.14 \pm 0.06(3)^{(a)}$ & $+0.03 \pm 0.07(3)^{(a)}$ \\
GCE corrected ${ }^{(b)}$ & $\ldots$ & $\ldots$ & $\ldots$ & $-0.10 \pm 0.04(3)$ & $-0.13 \pm 0.06(3)^{(a)}$ & $+0.03 \pm 0.07(3)^{(a)}$ \\
\hline N97 & $+0.15 \pm 0.05(1)$ & $+0.14 \pm 0.05(1)$ & $+0.01 \pm 0.08(1)$ & $-0.10 \pm 0.06(1)$ & $-0.10 \pm 0.06(1)$ & $+0.00 \pm 0.09(1)$ \\
K02 & $\ldots$ & $+0.11 \pm 0.08(1)$ & $\ldots$ & $\ldots$ & $-0.08 \pm 0.05(1)$ & $\ldots$ \\
G18 & $\ldots$ & $\ldots$ & $\ldots$ & $-0.13 \pm 0.14(3)$ & $-0.03 \pm 0.03(3)$ & $-0.10 \pm 0.15(3)$ \\
\hline
\end{tabular}

Notes. Our recommended values are the weighted average (by the inverse variance) of the results obtained using the Me14 and Re03 line lists. For the analysis based on the single Eu II feature in Re03 line list, we conservatively assumed $\sigma_{\text {int }}=0.05$ dex. The number in brackets gives the number of lines used. Keywords for literature studies are N97: Neuforge-Verheecke \& Magain (1997); K02: Koch \& Edvardsson (2002); G18: Guiglion et al. (2018). Note that the last study made use of archival HARPS spectra that are different from ours. ${ }^{(a)}$ These values are affected by likely underestimated abundances in $\alpha$ Cen B at the $\sim 0.05$ dex level (see Sect. 3). ${ }^{(b)}$ Computed from the [Eu/Fe]-age relation of Bedell et al. (2018) (see Sect. 4.1 for details). The uncertainty in the slope of the relation was propagated to the abundances.

through a differential analysis. As discussed in Sect. 2, nonLTE effects in the Sun are very small for Eu II. Although a detailed quantitative investigation is warranted, we thus do not expect differential corrections to significantly bias our results. On the other hand, Mucciarelli et al. (2008) predicted negligible 3D corrections for Eu II $\lambda 6645$ for stars with parameters that are representative of those of $\alpha$ Cen AB. The accuracy of our abundance results strongly depends on the reliability of the stellar parameters assumed. For both stars, our $T_{\text {eff }}$ is compatible within the uncertainty with the value based on the interferometric measurements (Kervella et al. 2017a) with VLTI//PIONIER (the Very Large Telescope Interferometer equipped with Precision Integrated-Optics Near-infrared Imaging ExpeRiment). However, the strength of the Eu II lines is particularly sensitive to $\log g$. Although our spectroscopic value for $\alpha$ Cen A is indistinguishable from the accurate asteroseismic estimate, $\log g$ for $\alpha$ Cen B appears to be underestimated by 0.09 and 0.23 dex using the Re03 and Me14 line lists, respectively (see M18). We regard this bias as the most significant source of systematic error because it likely leads to an underestimation of $[\mathrm{Eu} / \mathrm{H}]$ and $[\mathrm{Eu} / \mathrm{Fe}]$ in this star by $\sim 0.05 \mathrm{dex}$.

\section{Discussion}

\subsection{Comparison with previous results of europium abundances in $\alpha$ Cen $A B$}

Our abundances agree well with previous estimates reported in the literature (Table 2), but our study is generally based on more lines and benefits from higher quality spectroscopic and HFS data. We do not discuss the results of Allende Prieto et al. (2004) because there is evidence that their $T_{\text {eff }}$ scale is too cool (see discussion in M18).

To summarise our abundance analysis results, we find that europium is depleted with respect to iron in $\alpha$ Cen $\mathrm{AB}$ by $\sim 0.1$ dex compared to the Sun and that there is a lack of evidence for a different Eu content in the two components. Our study does not support the claim that the $[\mathrm{Eu} / \mathrm{Fe}]$ values differ by as much as 0.16 dex between the two stars (Hinkel \& Kane 2013), but we note that Allende Prieto et al. (2004) is their sole literature source for $\mathrm{Eu}$. Our conclusions are still valid if the slight underestimation of the abundances in $\alpha$ Cen B discussed above is taken into account. Generally speaking, we expect the abundances determined through our differential analysis to be more accurate for $\alpha$ Cen A because in view of the similarity with the solar parameters, they are much less sensitive to deficiencies in the modelling of the atmosphere or non-LTE, 3D, and atomic diffusion effects. This caveat for the abundances of $\alpha$ Cen B also applies to other differential studies in the literature (e.g. Guiglion et al. 2018).

The exact production sites of europium are still uncertain. Cataclysmic events, such as compact binary mergers or core-collapse supernovae, are proposed to play a role in the nucleosynthesis of Eu, although their relative importance is debated (Skúladóttir et al. 2019, and references therein). The $[\mathrm{Eu} / \mathrm{Fe}]$ ratios are weakly modulated by Galactic chemical evolution (GCE) effects; the dependence as a function of stellar age appears to be nearly flat (Bedell et al. 2018). Assuming an age for $\alpha$ Cen $\mathrm{AB}$ of $6 \pm 1 \mathrm{Ga}$ (M18, and references therein) and the linear relations between $[\mathrm{Eu} / \mathrm{Fe}]$ and age for solar analogues of Bedell et al. (2018), we attribute only $\sim 0.01$ dex of depletion to GCE (Table 2). Similar GCE effects are also suggested for metal-rich stars (Delgado Mena et al. 2019).

\subsection{Comparison of europium abundances between $\alpha$ Cen $A B$ and other FGK stars}

In Fig. 2 (upper panel), we compare our [Eu/Fe] abundances of $\alpha$ Cen $\mathrm{AB}$ as a function of $[\mathrm{Fe} / \mathrm{H}]$ to the values for about 570 FGK dwarfs (Delgado Mena et al. 2019). Both $\alpha$ Cen A and $B$ appear at the high and low ends of the $[\mathrm{Fe} / \mathrm{H}]$ and $[\mathrm{Eu} / \mathrm{Fe}]$ distributions, respectively, demonstrating their unusual position in the $[\mathrm{Fe} / \mathrm{H}]-[\mathrm{Eu} / \mathrm{Fe}]$ locus defined by nearby, thin-disc FGK dwarfs. This conclusion may be sensitive to the (unavoidable) presence of zero-point abundance offsets between our study and Delgado Mena et al. (2019). However, Fig. 2 (lower panel) shows that the same picture holds when we consider the separate study of Battistini \& Bensby (2016). This indicates that such offsets are unlikely to be much larger than our abundance uncertainties. Hence, the somewhat peculiar position of $\alpha \mathrm{Cen} \mathrm{AB}$ on the $[\mathrm{Fe} / \mathrm{H}]-[\mathrm{Eu} / \mathrm{Fe}]$ locus is most likely a reflection of their true nature.

If we had used another major rock-forming element and not $\mathrm{Fe}$ as reference (e.g. Si; Unterborn et al. 2015), our conclusions would not have been qualitatively modified because their 


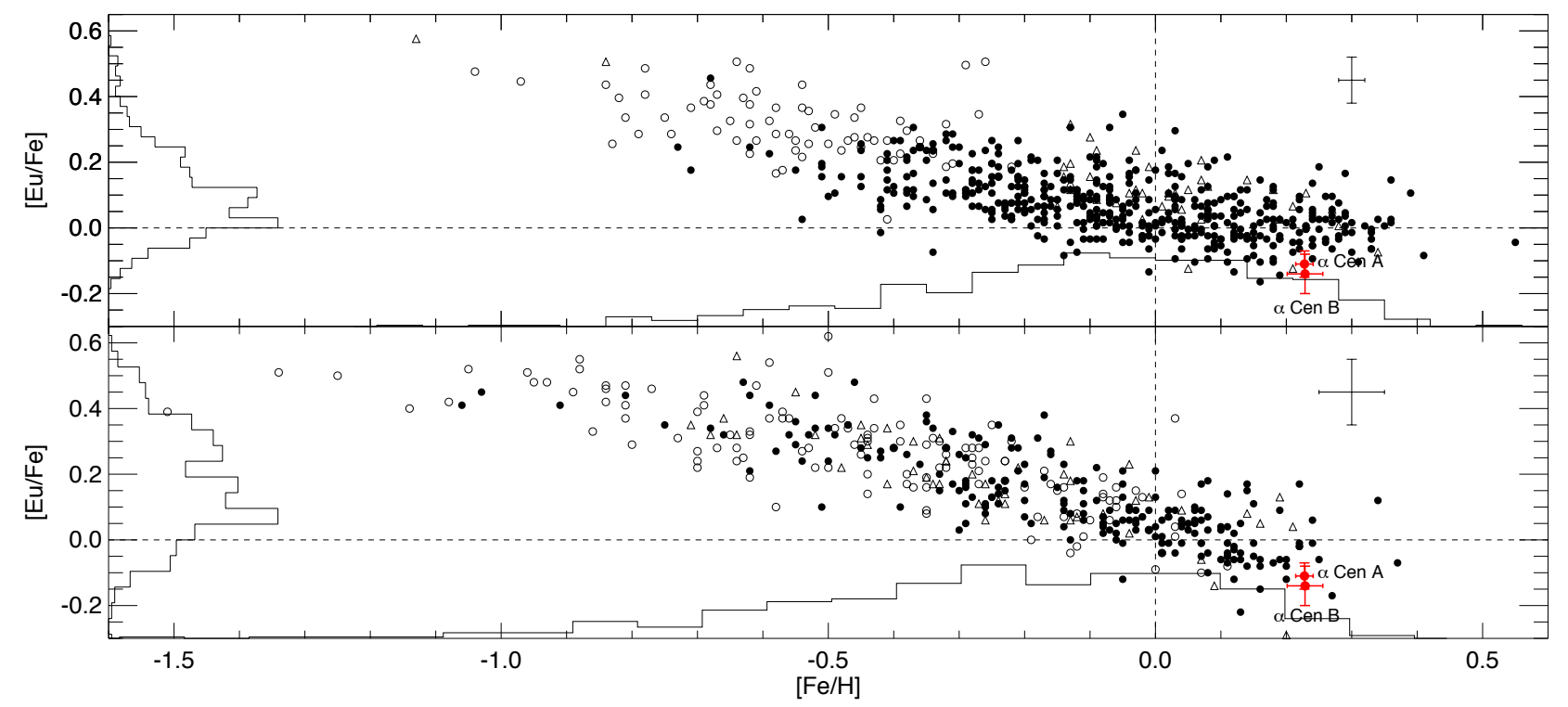

Fig. 2. Our abundance results for $\alpha$ Cen $\mathrm{A}$ and B overlaid on the $[\mathrm{Eu} / \mathrm{Fe}]-[\mathrm{Fe} / \mathrm{H}]$ diagram for large samples of Sun-like stars in the literature. Upper panel: data for about 570 FGK stars that have available Eu abundances in Delgado Mena et al. (2017, 2019). The [Eu/Fe] abundances are taken from Delgado Mena et al. (2017), but were shifted upwards by 0.016 dex, as recommended by Delgado Mena et al. (2019). Lower panel: same as the upper panel, but with data for the 380 FG stars that have available Eu abundances in Battistini \& Bensby (2016). The typical uncertainties of the two comparison datasets are shown in the upper right corner of the respective panel. Stars from thin- and thick-disc populations are shown as filled and open circles, respectively, while other stellar populations (i.e. halo, bulge, and uncertain thin-/thick-disc stars) are indicated as open triangles. The histograms on the $x$-axis and $y$-axis show the respective distributions of $[\mathrm{Fe} / \mathrm{H}]$ and $[\mathrm{Eu} / \mathrm{Fe}]$ of these $\mathrm{FG}(\mathrm{K})$ stars. The horizontal and vertical dashed lines indicate the zero-points of the diagrams (solar).

abundances are tightly correlated with that of iron in thin-disc stars (e.g. Bitsch \& Battistini 2020). As shown by the [Eu/Si][Fe/Si] diagram (Fig. 3), $\alpha$ Cen AB still lies in the lower tail of the $[\mathrm{Eu} / \mathrm{Si}]$ distribution for the sample of thin-disc stars of Delgado Mena et al. (2019). Specifically, [Eu/Si] in $\alpha$ Cen AB is depleted by $\sim 0.15$ dex compared to the Sun. However, $\alpha$ Cen $\mathrm{AB}$ appears to be not a peculiar, but an average case, among these comparison stars in terms of [Fe/Si], which alludes to the first-order, planetary internal structure (Wang et al. 2019b).

\subsection{Implications for radiogenic heat budgets in putative $\alpha$-Cen-Earths}

To some extent, we can explore implications from the peculiar features of $\alpha \mathrm{Cen} \mathrm{AB}$ on the distributions of [Eu/Fe] and [Eu/Si] for radiogenic heat production in putative terrestrial-like planets in the system (i.e. " $\alpha$-Cen-Earth").

First of all, because the binary stars have a common origin and their $[\mathrm{Eu} / \mathrm{Si}]$ and $[\mathrm{Fe} / \mathrm{Si}]$ abundances overlap (shown in Fig. 3), we did not distinguish such a putative planet around A or $\mathrm{B}$, but computed the weighted average of the $\mathrm{Eu}$ abundances in $\mathrm{A}$ and B to construct an average $\alpha$-Cen-Earth of the system.

Second, we converted the $\mathrm{Eu}$ abundance into the abundances of the $r$-process radioactive elements $T h$ and $U$ (we discuss $\mathrm{K}$ separately). We adopted the observational average $(0.014 \pm 0.045 \mathrm{dex})$ of $[\mathrm{Th} / \mathrm{Eu}]$ values of the solar analogues on the zero-age main sequence, ZAMS (Botelho et al. 2019) to first compute Th abundance from Eu. Then the nearly constant ${ }^{238} \mathrm{U} / \mathrm{Th}$ production ratio $\left(0.571_{-0.031}^{+0.037}\right)$ in meteorites and Galactic halo stars (Dauphas 2005) was adopted to obtain the abundance of ${ }^{238} \mathrm{U}$. Although the isotopic ratio ${ }^{235} \mathrm{U} /{ }^{238} \mathrm{U}$ may not be necessarily constant during the Galactic history, its variance, however, is limited when we consider the time interval between 6 and $9 \mathrm{Ga}$ of the Galactic evolution (Frank et al. 2014); within this interval,

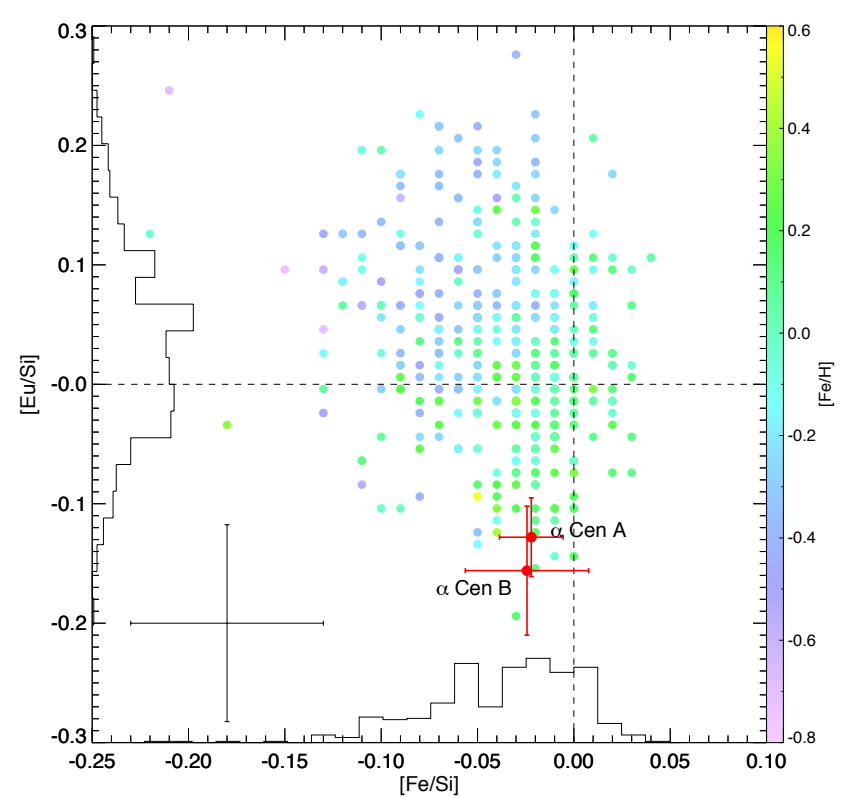

Fig. 3. Our abundance results for $\alpha$ Cen $\mathrm{A}$ and $\mathrm{B}$ overlaid on the [Eu/Si]-[Fe/Si] diagram for the sample of thin-disc stars (colour-coded as a function of $[\mathrm{Fe} / \mathrm{H}]$ ) of Delgado Mena et al. (2019). Their typical uncertainties are indicated in the bottom left corner. The histograms on the $x$-axis and $y$-axis show the respective distributions of $[\mathrm{Fe} / \mathrm{Si}]$ and $[\mathrm{Eu} / \mathrm{Si}]$ of these thin-disc stars. The horizontal and vertical dashed lines indicate the zero-points of the diagram (solar).

the Solar System and the $\alpha$ Cen system formed. We therefore preferred to take a simple approach and adopted the well-known initial Solar System ${ }^{235} \mathrm{U} /{ }^{238} \mathrm{U}(24.286 / 75.712$; Lodders et al. 2009), set for $4.56 \mathrm{Ga}$ ago, to infer the abundance of ${ }^{235} \mathrm{U}$ for 
$\alpha$ Cen AB. This is admittedly a crude simplification, and we discuss this caveat further in the subsequent section.

Third, because both $\mathrm{U}$ and Th are refractory lithophile elements (RLEs), their stellar abundances upon normalising to a major rock-forming $\mathrm{RLE}$ (e.g. $\mathrm{Mg}$ or $\mathrm{Si}$ ) implicitly reflect their concentrations in the primitive mantle ${ }^{4}$ of a rocky planet. We preferred $\mathrm{Mg}$ over $\mathrm{Si}$ because $\mathrm{Si}$ is also widely recognised as a major light element in the core in spite of its primary nature as a lithophile (Wang et al. 2018 and references therein). On this basis, we obtained the mass ratio of each individual radionuclide (X) to $\mathrm{Mg}$ (i.e. X/Mg). Independently, we calculated X/Mg of the (primitive) mantle of the Earth based on the literature. With the known mantle concentrations (by mass) of individual radionuclides in the Earth (see Table A.1), we scaled them by the relative $\mathrm{X} / \mathrm{Mg}$ between $\alpha$-Cen(-Earth) and the Earth to obtain the corresponding nuclide concentrations in the primitive mantle of an $\alpha$-Cen-Earth. For simplicity, the mantle mass of $\alpha$ Cen Earth was assumed to be equal to that of the Earth (this assumption is further discussed in Sect. 4.4).

Furthermore, based on the radioactive decay and their known half-lives (Turcotte \& Schubert 2002) as well as based on the age of the $\alpha$ Cen AB system $(6 \pm 1 \mathrm{Ga}$; M18), we modelled the radionuclide concentrations in the mantle of such an $\alpha$-Cen-Earth over geological time. For normalisation purposes, the concentration of the stable and major rock-forming element $\mathrm{Mg}$ in the mantle was kept unchanged.

With the heat generation rates (per unit mass of the individual radionuclides; Dye 2012), we finally computed the heat output for both the nascent and present-day $\alpha$-Cen-Earth. We summarise our model constraints (with references) as well as the concentration and radiogenic heating estimates in Table 3. We also report our corresponding calculations for the Earth in Table A.1 for comparison.

Now, we discuss ${ }^{40} \mathrm{~K}$, which we did not directly constrain from $\mathrm{Eu}$. We instead based it on a GCE assumption for cosmochemically Earth-like planets (Frank et al. 2014).

Potassium is not only irrelevant to $\mathrm{Eu}$ (in terms of abundances) because of its distinct production and destruction pathways (Clayton 2003; Zhang et al. 2006), but also is a volatile element, which means that its abundance in a planet-hosting star cannot be directly reflected in a rocky planet (because of devolatilisation during planet formation; Wang 2018, and references therein). Simultaneously, potassium is one of the few most challenging (rock-forming) elements to be widely/accurately measured in the photospheres of stars because only the strong $\mathrm{K} I$ $7698.96 \AA$ line, which is difficult to model properly and strongly affected by non-LTE effects, can be measured (Reggiani et al. 2019; Takeda 2019). Potassium is also not covered by most spectrographs (e.g. HARPS). Therefore we are at present forced to make crude assumptions about the abundance of $\mathrm{K}$, or more specifically, of ${ }^{40} \mathrm{~K}$. In light of the starting mantle concentrations of ${ }^{40} \mathrm{~K}\left(C\left({ }^{40} \mathrm{~K}\right)\right)$ for cosmochemically Earth-like planets (i.e. assuming their volatile depletion scales relative to their host stars to be the same as that of the Earth to the Sun) as a function of the time after galaxy formation (Frank et al. 2014), we note that the variance in $C\left({ }^{40} \mathrm{~K}\right)$ is insignificant within about 6-9 Ga into the Galactic history. We therefore directly translated the concentration of ${ }^{40} \mathrm{~K}$ in the primitive mantle of an $\alpha$-CenEarth from that of the primitive mantle of the early Hadean Earth (4.56 Ga ago). For the instantaneous radionuclide concentrations and heat production (due to the radioactive decay), the same

$\overline{4}$ Primitive mantle means the present-day mantle plus the crust.
Table 3. Model quantities and results of mantle concentrations and heat output of radionuclides in a putative $\alpha$-Cen-Earth.

\begin{tabular}{|c|c|c|c|}
\hline Constraint & Value & \multicolumn{2}{|c|}{ Reference } \\
\hline$[\mathrm{Eu} / \mathrm{Mg}]^{(a)}$ & $-0.152 \pm 0.047$ & \multicolumn{2}{|c|}{ This work \& M18 } \\
\hline$[\mathrm{Th} / \mathrm{Eu}]^{(b)}$ & $0.014 \pm 0.045$ & \multicolumn{2}{|c|}{ Botelho et al. (2019) } \\
\hline${ }^{238} \mathrm{U} / \mathrm{Th}$ & $0.571_{-0.031}^{+0.037}$ & \multicolumn{2}{|c|}{ Dauphas (2005) } \\
\hline${ }^{235} \mathrm{U} / 238 \mathrm{U}^{(c)}$ & $24.286 / 75.712$ & \multicolumn{2}{|c|}{ Lodders et al. (2009) \& Frank et al. (2014) } \\
\hline$M_{\text {Mantle }}\left(10^{24} \mathrm{~kg}\right)$ & $4.0312 \pm 0.0179$ & \multicolumn{2}{|c|}{ Wang et al. (2018) } \\
\hline & & \multicolumn{2}{|c|}{ Mantle concentration (ppb) } \\
\hline Nuclide (X) & $\mathrm{X} / \mathrm{Mg}^{(d)}$ & Upon formation $^{(e)}$ & Present-day ${ }^{(f)}$ \\
\hline${ }^{232} \mathrm{Th}$ & $1.95_{-0.48}^{+0.64} \times 10^{-7}$ & $43.4_{-10.7}^{+14.2}$ & $32.2_{-8.0}^{+10.5}$ \\
\hline${ }^{235} \mathrm{U}$ & $3.61_{-0.91}^{+1.2} \times 10^{-8}$ & $8.1_{-2.0}^{+2.7}$ & $0.02 \pm 0.01$ \\
\hline${ }^{238} \mathrm{U}$ & $1.14_{-0.29}^{+0.38} \times 10^{-7}$ & $9.7_{-2.5}^{+3.3}$ & $10.0_{-2.5}^{+3.3}$ \\
\hline \multirow[t]{2}{*}{${ }^{40} \mathrm{~K}$} & $\ldots$ & $353.6 \pm 37.3^{(g)}$ & $12.7 \pm 1.3^{(g)}$ \\
\hline & & \multicolumn{2}{|c|}{ Heat (TW) } \\
\hline Nuclide (X) & $h\left(\mathrm{~W} \mathrm{~kg}^{-1}\right)^{(h)}$ & Upon formation ${ }^{(i)}$ & Present-day ${ }^{(i)}$ \\
\hline${ }^{232} \mathrm{Th}$ & $2.628 \times 10^{-5}$ & $4.6_{-1.1}^{+1.5}$ & $3.4_{-0.8}^{+1.1}$ \\
\hline${ }^{235} \mathrm{U}$ & $5.6847 \times 10^{-4}$ & $18.5_{-4.7}^{+6.2}$ & $0.05_{-0.01}^{+0.02}$ \\
\hline${ }^{238} \mathrm{U}$ & $9.513 \times 10^{-5}$ & $9.7_{-2.5}^{+3.3}$ & $3.8_{-1.0}^{+1.3}$ \\
\hline${ }^{40} \mathrm{~K}$ & $2.847 \times 10^{-5}$ & $12.7 \pm 1.3$ & $1.5 \pm 0.2$ \\
\hline Total & $\ldots$ & $73.4_{-6.9}^{+8.3}$ & $8.8_{-1.3}^{+1.7}$ \\
\hline
\end{tabular}

Notes. ${ }^{(a)}[\mathrm{Eu} / \mathrm{Mg}]($ dex $)$ is calculated from $[\mathrm{Eu} / \mathrm{Fe}]-[\mathrm{Mg} / \mathrm{Fe}]$, where $[\mathrm{Mg} / \mathrm{Fe}]$ comes from the weighted average of the results in M18 obtained using the Me14 and Re03 line lists. ${ }^{(b)} 100 \%$ of Th is ${ }^{232} \mathrm{Th}$. ${ }^{(c)}$ As explained in the text, ${ }^{235} \mathrm{U} /{ }^{238} \mathrm{U}$ refers to the Solar System value at the time of its formation (Lodders et al. 2009) based on the limited variance of the ratio within 6-9 Ga into the Galactic history (Frank et al. 2014). ${ }^{(d)} \mathrm{X} / \mathrm{Mg}$ is a mass ratio. While converting differential values (e.g. $\left[{ }^{232} \mathrm{Th} / \mathrm{Mg}\right]=\left[{ }^{232} \mathrm{Th} / \mathrm{Eu}\right]-[\mathrm{Eu} / \mathrm{Mg}]$, in dex) into the mass ratios (e.g. ${ }^{232} \mathrm{Th} / \mathrm{Mg}$ ) in a linear scale, the reference solar abundances come from Asplund et al. (2009) and the atomic and isotopic masses are takent from Wieser et al. (2013) and Audi \& Wapstra (1993), respectively. ${ }^{(e)}$ See text. $C_{0}(\alpha$-Cen-Earth $)=\frac{\mathrm{X} / \mathrm{Mg}(\alpha-\text { Cen-Earth })}{\mathrm{X} / \mathrm{Mg}(\text { Earth })} \times C_{0}($ Earth $)$, where $C_{0}$ represents the mantle concentration of an individual radionuclide upon the planet formation; X/Mg ( $\alpha$-Cen-Earth) refers to Col. 2 in panel 2 of this table $\left({ }^{40} \mathrm{~K}\right.$ is excluded from this calculation); $\mathrm{X} / \mathrm{Mg}$ (Earth) refers to Table A.1 (for normalisation purposes, the $\mathrm{Mg}$ concentration was kept unchanged over geological time). ${ }^{(f)}$ See text. $C_{t}=C_{0} \times \mathrm{e}^{-t \ln 2 / T_{1 / 2}}$, where $t$ represents the system age (the current age of $\alpha \mathrm{Cen} \mathrm{AB}$ is $6 \pm 1 \mathrm{Ga}$ as of M18); $T_{1 / 2}$ are the half-lives of these radionuclides $\left({ }^{232} \mathrm{Th}-14.0 \mathrm{Ga} ;{ }^{235} \mathrm{U}-0.704 \mathrm{Ga}\right.$; ${ }^{238} \mathrm{U}-4.47 \mathrm{Ga} ;{ }^{40} \mathrm{~K}-1.25 \mathrm{Ga}$; Turcotte \& Schubert 2002). ${ }^{(g)}$ See text. Concentration of ${ }^{40} \mathrm{~K}$ upon planet formation, $C_{0}\left({ }^{40} \mathrm{~K}\right)$, is supplemented by the starting mantle concentration of ${ }^{40} \mathrm{~K}$ in the Earth upon its formation, according to the GCE model for cosmochemically Earth-like planets (Frank et al. 2014); the present-day concentration of ${ }^{40} \mathrm{~K}$ is calculated based on the radioactive decay process following the equation in footnote $(f) .{ }^{(h)} h$ (the rate of heating per unit mass of a radionuclide) is taken from Dye (2012). ${ }^{(i)}$ Calculated by multiplying the corresponding concentrations (upon planet formation and at the present day) with the heat generation rates, $h$, of individual radionuclides.

processes mentioned earlier apply (see more details in Table 3 and footnotes therein).

Figure 4 illustrates the calculations of both mantle concentrations and heat output of individual radionuclides $\left({ }^{232} \mathrm{Th},{ }^{235} \mathrm{U}\right.$, ${ }^{238} \mathrm{U}$, and ${ }^{40} \mathrm{~K}$ ) in a putative $\alpha$-Cen-Earth over geological time (logically presumed equal to the system age). In terms of the mantle concentrations, ${ }^{40} \mathrm{~K}$ is the most abundant heat-producing nuclide since planet formation until about the age of $4 \mathrm{Ga}$, 


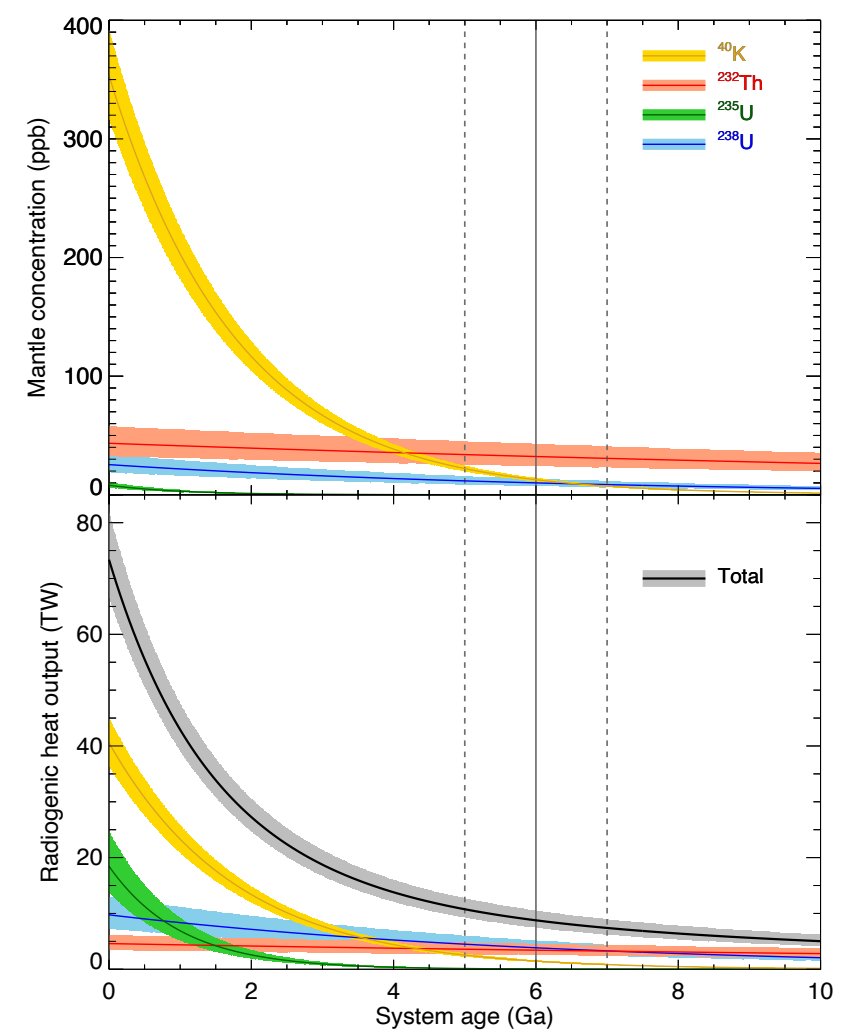

Fig. 4. Estimated mantle concentrations (upper panel) and radiogenic heat output (lower panel) of ${ }^{40} \mathrm{~K},{ }^{232} \mathrm{Th},{ }^{235} \mathrm{U}$, and ${ }^{238} \mathrm{U}$ in an $\alpha$-CenEarth as a function of the system age. The wedge around each curve represents the uncertainties of the estimates over time. The solid and dashed vertical lines indicate the current age $(6 \pm 1 \mathrm{Ga}$; Morel 2018) of the $\alpha$ Cen AB system.

when ${ }^{232} \mathrm{Th}$ becomes dominant. ${ }^{238} \mathrm{U}$ is relatively modest over geological time, while ${ }^{235} \mathrm{U}$ has become negligible since about 1.5-2 Ga. These trends in concentrations are not exactly identical to those in the heat output of these radionuclides because their heat generation rates are different. Prominently, ${ }^{235} \mathrm{U}$ contributed a significant amount of heating (only a factor of 2 lower than the highest contributor $-{ }^{40} \mathrm{~K}$ ) at planet formation and only became negligible since about $4 \mathrm{Ga} .{ }^{238} \mathrm{U}$ has started to play the most significant role in heat production from about the age of $3.5 \mathrm{Ga}$, when ${ }^{40} \mathrm{~K}$ underwent its demise and then was further subordinated by ${ }^{232} \mathrm{Th}$ by the time the system reached $4.5 \mathrm{Ga}$. Thereafter, ${ }^{238} \mathrm{U}$ and ${ }^{232} \mathrm{Th}$ dominated the radiogenic heat output by contributing approximately equally up to about $10 \mathrm{Ga}$, which is the maximum time we modelled, with ${ }^{232} \mathrm{Th}$ gradually taking over heat production in all old terrestrial-type planets (Frank et al. 2014) because it has the longest half-life (14 Ga).

In comparison with the total radiogenic heat output of the Earth (see Tables 3 and A.1), an $\alpha$-Cen-Earth would have generated $73.4_{-6.9}^{+8.3} \mathrm{TW}$ at the time of formation and $8.8_{-1.3}^{+1.7} \mathrm{TW}$ at the present day. We note that this is $23 \pm 5 \%$ and $54 \pm 5 \%$ lower than estimated for the Hadean Earth $(94.9 \pm 5.5$ TW) and for the modern Earth $(19.0 \pm 1.1 \mathrm{TW})$. In our gedankenexperiment, an $\alpha$-Cen-Earth is intrinsically less geologically active than the Earth, overall.

\subsection{Uncertainty/limitation analysis}

Our reported uncertainties associated with the radionuclide concentrations and heat output mainly come from the uncertainties on the stellar abundances ( $\mathrm{Eu}$ and the reference element $\mathrm{Mg}$ ), those associated with our model constraints, the uncertainties on the mantle concentrations of the Earth, and the uncertainty of the stellar age. The uncertainties of heat output from the individual radionuclides are added in quadrature to estimate the uncertainty of the total heat output. It is noteworthy that uncertainties in a relative sense are smaller than those in absolute values. For example, the comparison of heat output between an $\alpha$-Cen-Earth and the Earth has yielded smaller error bars on their relative differences $(23 \pm 5 \%$ and $54 \pm 5 \%)$ than those on the absolute heat output values $\left(73.4_{-6.9}^{+8.3}\right.$ and $\left.8.8_{-1.3}^{+1.7}\right)$ of such an $\alpha$-Cen-Earth because in the latter case, the uncertainties on the reference (the Earth) have also been included.

The accuracy of our model rests on the robustness of our assumptions. We adopted the constant values from Botelho et al. (2019) and Dauphas (2005) for [Th/Eu] and $\left[{ }^{238} \mathrm{U} / \mathrm{Th}\right]$, respectively. We used a constant value for $[\mathrm{Th} / \mathrm{Eu}]$ following Botelho et al. (2019). However, as we noted earlier, the thorium abundance is determined through modelling a single line that is also heavily blended with other elemental lines, limiting the accuracy of the modelled abundance regardless of the applied treatments. Similar concerns also apply to the U/Th ratio from Dauphas (2005), which may be further affected by the representativeness of the dominant sample of halo stars chosen (even though meteoritic data have also been considered) for the solar-like systems. We emphasise that such measurements/modellings on long-lived radionuclides are, to our knowledge, the best available. For ${ }^{235} \mathrm{U} /{ }^{238} \mathrm{U}$, it may be more appropriate to run GCE corrections on the adopted early Solar System value (24.286/75.712; Lodders et al. 2009), despite our observation of its limited variance for stars like the Sun and $\alpha$ Cen AB that were formed 6-9 Ga into the Galactic history (Frank et al. 2014). In a more recent work on GCE, Côté et al. (2019) reported an unusually large error bar $(60 \%)$ on the initial Solar System ${ }^{235} \mathrm{U} /{ }^{238} \mathrm{U}$ ratio. If we incorporate this into our calculations, it would have an effect on our reported error bars for the starting concentration and heat output of ${ }^{235} \mathrm{U}$ by a factor of about 2 , which is not significant for the total heat output over geological time, however.

In addition, because we have adopted the concept "cosmochemically Earth-like planets" (Frank et al. 2014) for considering ${ }^{40} \mathrm{~K}$, our calculations of radiogenic heating from ${ }^{40} \mathrm{~K}$ are applicable only to habitable-zone terrestrial-like planets. These are assumed to receive the equivalent irradiation from their parent stars as the Earth from the Sun (Hart 1979). When we consider that planet formation processes may alter the primordial devolatilisation scale controlled by evaporation/condensation out of a nebular gas (Grossman \& Larimer 1974; O'Neill \& Palme 2008; Albarède 2009; Hin et al. 2017; Norris \& Wood 2017; Wang et al. 2019a; Fegley et al. 2020), such planets might be expected to have shared similar formation histories as our Earth's, at least in terms of the final effect on the volatile depletion. For this reason, we also caution that it is premature to extend our conclusions to the planet(s) orbiting the M5 red dwarf Proxima Cen (Anglada-Escudé et al. 2016; Damasso et al. 2020) in view of the still-debated origin of this star (e.g. Kervella et al. 2017b; Beech et al. 2017; Feng \& Jones 2018) and because the transposition of the stellar abundances to planet bulk composition might be dramatically different between Sun-like stars and the chromospherically active, flaring M stars.

Finally, we note that our assumption of the equal mantle mass of a putative $\alpha$-Cen-Earth as that of the Earth is out of convenience only to enable us to focus on the discussion of the heat output dictated by the estimated mantle concentrations of these radionuclides and then make comparisons between the two 
planets. It is noteworthy that the current detection limits of radial velocity are still far from being resolvable to any Earth-mass, habitable-zone planet: $M \sin i \sim 50 M_{\oplus}$ for $\alpha$ Cen A and $\sim 8 M_{\oplus}$ for $\alpha$ Cen B (Zhao et al. 2018). On the other hand, the mantle/core ratios between an $\alpha$-Cen-Earth and the Earth should not be significantly different, in view of the overlapped [Fe/Si] values between $\alpha$ Cen $\mathrm{AB}$ and the Sun (Fig. 3). However, we do not proceed here to assess how the planetary mass and mantleto-core ratio would affect the radiogenic heat budget; instead, we refer to Frank et al. (2014; in particular, their Sect. 5.2 and Fig. 13) for a detailed discussion of this aspect.

\section{Conclusions}

In the context of studying the potential of $\mathrm{Eu}$ as a convenient tool for diagnosing exoplanetary radiogenic heat power (in the absence of abundance measurements of long-lived radionuclides in the majority of planet hosting stars), we present a detailed determination of the abundances of this element in the photospheres of $\alpha$ Cen AB, our nearest Sun-like stars. Our spectroscopic analysis shows that europium is depleted with respect to iron by $\sim 0.1 \mathrm{dex}(\sim 25 \%)$ and to silicon by $\sim 0.15 \operatorname{dex}(\sim 40 \%)$ compared to solar in the two binary components. A comparison with a large sample of FGK stars shows that this depletion in $\alpha$ Cen $\mathrm{AB}$ appears to be true relative to the majority of these Sun-like stars. This may have important implications for the potential of an $\alpha$-Cen-Earth (a putative terrestrial-like planet in the system) in generating radiogenic heating if our view on using $\mathrm{Eu}$ as a proxy for long-lived radionuclides $\left({ }^{232} \mathrm{Th},{ }^{235} \mathrm{U}\right.$, and ${ }^{238} \mathrm{U}$ ) is correct. ${ }^{40} \mathrm{~K}$ has to be treated independently because its nucleosynthesis pathways are different and its behaviour is volatile.

We have applied a simple and intuitive approach to quantify the radiogenic heat output propagated from the Eu abundances combined with other assumptions, including a GCE model for ${ }^{40} \mathrm{~K}$. Our first-order estimates lead us to propose that the radiogenic heat budget in an $\alpha$-Cen-Earth is $73.4_{-6.9}^{+8.3} \mathrm{TW}$ upon its formation and $8.8_{-1.3}^{+1.7} \mathrm{TW}$ at the present day, $23 \pm 5 \%$ and $54 \pm 5 \%$ lower than that in the Hadean Earth $(94.9 \pm 5.5 \mathrm{TW})$ and in the modern Earth (19.0 \pm 1.1 TW), respectively. If we assume all other conditions, especially the primordial gravitational energy (as yet unconstrained), are not significantly different between the $\alpha$-Cen-Earth and our Earth, the mantle convection in the $\alpha$ Cen-Earth would be comparably weaker than our planet over its equivalent evolution history (as the Earth's), subduing its geological activity and by extension, its long-duration habitable potential.

The multivariate nature of planetary evolution is a complex process (Stevenson 2004), but the similar [Fe/Si] ratios between $\alpha$ Cen $\mathrm{AB}$ and the Sun (shown in Fig. 3) reveal to us at first order that the relative core-to-mantle mass fractions are probably not so different between an $\alpha$-Cen-Earth and the Earth. Detailed modelling of bulk compositions and internal structures of such $\alpha$-Cen-Earths will be investigated in our subsequent paper.

In short, we conclude with caution that Eu can be a convenient and practical tool, along with other constraints, in helping understand the exoplanetary radiogenic heating potential. This may apply to a population analysis of this aspect for increasingly more discovered rocky exoplanets, of which the host stellar abundances for long-lived radionuclides can rarely be measured so far.

Acknowledgements. We thank an anonymous referee for his/her swift and insightful comments. We also thank D. J. Stevenson for useful comments on an earlier version of the manuscript and C. H. Lineweaver for early discussion. This work has been carried out within the framework of the National Centre of Competence in Research PlanetS supported by the Swiss National Science Foundation. H.S.W and S.P.Q acknowledge the financial support of the SNSF. T.M. acknowledges financial support from Belspo for contract PRODEX PLATO mission development. S.J.M. thanks the Collaborative for Research in Origins (CRiO) at the University of Colorado, which was supported by The John Templeton Foundation (PI: S. Benner/FfAME): the opinions expressed in this publication are those of the authors and do not necessarily reflect the views of the John Templeton Foundation. S.J.M. also acknowledges the NASA Solar System Workings Program, grant No. 80NSSC17K0732 (PI: O. Abramov/PSI). This work has made use of the VALD database, operated at Uppsala University, the Institute of Astronomy RAS in Moscow, and the University of Vienna. This research made use of NASA's Astrophysics Data System Bibliographic Services and the SIMBAD database operated at CDS, Strasbourg (France).

\section{References}

Albarède, F. 2009, Nature, 461, 1227

Allende Prieto, C., Barklem, P. S., Lambert, D. L., \& Cunha, K. 2004, A\&A, 420, 183

Andrade-Ines, E., \& Michtchenko, T. A. 2014, MNRAS, 444, 2167

Anglada-Escudé, G., Amado, P. J., Barnes, J., et al. 2016, Nature, 536, 437 Asplund, M., Grevesse, N., Sauval, A. J., \& Scott, P. 2009, ARA\&A, 47, 481 Audi, G., \& Wapstra, A. 1993, Nucl. Phys. A, 565, 1

Battistini, C., \& Bensby, T. 2016, A\&A, 586, A49

Bazot, M., Bouchy, F., Kjeldsen, H., et al. 2007, A\&A, 470, 295

Bedell, M., Meléndez, J., Bean, J. L., et al. 2014, ApJ, 795, 23

Bedell, M., Bean, J. L., Meléndez, J., et al. 2018, ApJ, 865, 68

Beech, M., McCowan, C., \& Peltier, L. 2017, Am. J. Astron. Astrophys., 5, 1

Bisterzo, S., Travaglio, C., Gallino, R., Wiescher, M., \& Käppeler, F. 2014, ApJ, 787,10

Bisterzo, S., Travaglio, C., Wiescher, M., et al. 2016, J. Phys. Conf. Ser., 665

Bitsch, B., \& Battistini, C. 2020, A\&A, 633, A10

Bond, J. C., O'Brien, D. P., \& Lauretta, D. S. 2010, ApJ, 715, 1050

Botelho, R. B., Milone, A. d. C., Meléndez, J., et al. 2019, MNRAS, 482, 1690

Bruntt, H., Bedding, T. R., Quirion, P.-O., et al. 2010, MNRAS, 405, 1907

Chang, T. L., Qian, Q.-Y., Zhao, M.-T., \& Wang, J. 1994, Int. J. Mass Spectr. Ion Process., 139, 95

Clayton, D. D. 2003, Handbook of isotopes in the cosmos Hydrogen to gallium (Cambridge: Cambridge University Press)

Côté, B., Lugaro, M., Reifarth, R., et al. 2019, ApJ, 878, 156

Damasso, M., Del Sordo, F., Anglada-Escudé, G., et al. 2020, Sci. Adv., 6 , eaax 7467

Dauphas, N. 2005, Nature, 435, 1203

del Peloso, E. F., da Silva, L., \& Porto de Mello, G. F. 2005, A\&A, 434, 275

Delgado Mena, E., Tsantaki, M., Adibekyan, V. Z., et al. 2017, A\&A, 606, A94

Delgado Mena, E., Moya, A., Adibekyan, V., et al. 2019, A\&A, 624, A78

Doyle, A. E., Young, E. D., Klein, B., Zuckerman, B., \& Schlichting, H. E. 2019, Science, 366, 356

Dumusque, X., Pepe, F., Lovis, C., et al. 2012, Nature, 1, 4

Dye, S. T. 2012, Rev. Geophys., 50, 1

Fegley, B., Lodders, K., \& Jacobson, N. S. 2020, Chemie der Erde, 80, 125594

Feng, F., \& Jones, H. R. A. 2018, MNRAS, 473, 3185

Frank, E. a., Meyer, B. S., \& Mojzsis, S. J. 2014, Icarus, 243, 274

Gando, a., Gando, Y., Ichimura, K., et al. 2011, Nat. Geosci., 4, 647

Gray, D. F., Tycner, C., \& Brown, K. 2000, PASP, 112, 328

Grevesse, N., Asplund, M., \& Sauval, A. J. 2007, Space Sci. Rev., 130, 105

Grevesse, N., Scott, P., Asplund, M., \& Sauval, A. J. 2015, A\&A, 573, A27

Grossman, L., \& Larimer, J. W. 1974, Rev. Geophys., 12, 71

Guiglion, G., de Laverny, P., Recio-Blanco, A., \& Prantzos, N. 2018, A\&A, 619, A143

Gustafsson, B., Edvardsson, B., Eriksson, K., et al. 2008, A\&A, 486, 951

Hart, M. H. 1979, Icarus, 37, 351

Hatzes, A. P. 2013, ApJ, 770, 133

Heiter, U., Jofré, P., Gustafsson, B., et al. 2015, A\&A, 582, A49

Hin, R. C., Coath, C. D., Carter, P. J., et al. 2017, Nature, 549, 511

Hinkel, N. R., \& Kane, S. R. 2013, MNRAS, 432, L36

Hinkel, N. R., \& Unterborn, C. T. 2018, ApJ, 853, 83

Hinkel, N. R., Unterborn, C., Kane, S. R., Somers, G., \& Galvez, R. 2019, ApJ, 880,49

Ivans, I. I., Simmerer, J., Sneden, C., et al. 2006, ApJ, 645, 613

Jacobson, H. R., \& Friel, E. D. 2013, AJ, 145, 107

Ji, A. P., Frebel, A., Chiti, A., \& Simon, J. D. 2016, Nature, 531, 610

Kervella, P., Bigot, L., Gallenne, A., \& Thévenin, F. 2017a, A\&A, 597, A137

Kervella, P., Thévenin, F., \& Lovis, C. 2017b, A\&A, 598, L7 
Kite, E. S., Manga, M., \& Gaidos, E. 2009, ApJ, 700, 1732

Koch, A., \& Edvardsson, B. 2002, A\&A, 381, 500

Lawler, J. E., Wickliffe, M. E., den Hartog, E. A., \& Sneden, C. 2001, ApJ, 563, 1075

Lenardic, A., Cooper, C. M., \& Moresi, L. 2011, Phys. Earth Planet. Inter., 188, 127

Lichtenberg, T., Golabek, G. J., Gerya, T. V., \& Meyer, M. R. 2016, Icarus, 274, 350

Lingam, M., \& Loeb, A. 2020, ApJ, 889, L20

Liu, F., Yong, D., Asplund, M., et al. 2020, MNRAS, 495, 3961

Lodders, K. 2003, ApJ, 591, 1220

Lodders, K., Palme, H., \& Gail, H.-P. 2009, Landolt- Börnstein, New Ser. Vol VI/4B, ed. J. Trümper (Berlin: Springer-Verlag), 560

Lugaro, M., Ott, U., \& Kereszturi, Á. 2018, Prog. Part. Nucl. Phys., 102, 1

Lyubetskaya, T., \& Korenaga, J. 2007, J. Geophys. Res. Solid Earth, 112, 1

Mashonkina, L., \& Gehren, T. 2000, A\&A, 364, 249

Meléndez, J., Ramírez, I., Karakas, A. I., et al. 2014, ApJ, 791, 14

Mishenina, T., Kovtyukh, V., Soubiran, C., \& Adibekyan, V. Z. 2016, MNRAS, 462,1563

Morel, T. 2018, A\&A, 615, A172

Mucciarelli, A., Caffau, E., Freytag, B., Ludwig, H. G., \& Bonifacio, P. 2008, A\&A, 484, 841

Neuforge-Verheecke, C., \& Magain, P. 1997, A\&A, 328, 261

Nimmo, F. 2015, Thermal and Compositional Evolution of the Core (Amsterdam: Elsevier B.V.), 9, 201

Noack, L., Rivoldini, A., \& Van Hoolst, T. 2017, Phys. Earth Planet. Inter., 269, 40

Norris, C. A., \& Wood, B. J. 2017, Nature, 549, 507

O’Neill, H. S. C., \& Palme, H. 2008, Philos. Trans. A. Math. Phys. Eng. Sci., 366,4205

Pagano, M. 2014, PhD thesis, Arizona State University, USA

Pagel, B. E. J. 1989, in Evolutionary Phenomena in Galaxies, eds. J. E. Beckman,

\& B. E. J. Pagel (Cambridge: Cambridge University Press), 201

Peek, K. M. G. 2009, PASP, 121, 755

Quarles, B., \& Lissauer, J. J. 2016, AJ, 151, 111

Quarles, B., \& Lissauer, J. J. 2018, AJ, 155, 130

Rajpaul, V., Aigrain, S., \& Roberts, S. 2016, MNRAS, 456, L6
Reddy, B. E., Tomkin, J., Lambert, D. L., \& Allende Prieto, C. 2003, MNRAS, 340, 304

Reggiani, H., Amarsi, A. M., Lind, K., et al. 2019, A\&A, 627, A1

Roederer, I. U., Sakari, C. M., Placco, V. M., et al. 2018, ApJ, 865, 129

Santos, N. C., Adibekyan, V., Dorn, C., et al. 2017, A\&A, 608, A1

Schubert, G., Turcotte, D. L., \& Olson, P. 2001, Mantle Convection in the Earth and Planets (Cambridge: Cambridge University Press)

Schuler, S. C., Vaz, Z. A., Katime Santrich, O. J., et al. 2015, ApJ, 815, 5

Seales, J., \& Lenardic, A. 2020, ApJ, 893, 114

Shahar, A., Driscoll, P., Weinberger, A., \& Cody, G. 2019, Science, 364, 433

Simmerer, J., Sneden, C., Cowan, J. J., et al. 2004, ApJ, 617, 1091

Skúladóttir, Á., Hansen, C. J., Salvadori, S., \& Choplin, A. 2019, A\&A, 631, A171

Sleep, N. H. 2007, Treat. Geophys., 9, 145

Sneden, C. A. 1973, PhD thesis, The University of Texas at Austin, Austin

Sneden, C., Cowan, J. J., \& Gallino, R. 2008, ARA\&A, 46, 241

Stevenson, D. J. 2003, CR Geosci., 335, 99

Stevenson, D. 2004, Nature, 428, 476

Takeda, Y. 2019, SAG Stars Galaxies, 2, id.1

Turcotte, D. L., \& Schubert, G. 2002, Geodynamics (Cambridge: Cambridge University Press)

Unterborn, C. T., Johnson, J. A., \& Panero, W. R. 2015, ApJ, 806, 139

Wang, H. 2018, PhD thesis, Australian National University, Australia

Wang, H. S., Lineweaver, C. H., \& Ireland, T. R. 2018, Icarus, 299, 460

Wang, H. S., Lineweaver, C. H., \& Ireland, T. R. 2019a, Icarus, 328, 287

Wang, H. S., Liu, F., Ireland, T. R., et al. 2019b, MNRAS, 482, 2222

Wieser, M. E., Holden, N., Coplen, T. B., et al. 2013, Pure Appl. Chem., 85, 1047

Wilford, J. 2011, AUSGEO News, 101, 8

Wood, B. J., Smythe, D. J., \& Harrison, T. 2019, Am. Mineral., 104, 844

Yong, D., Karakas, A. I., Lambert, D. L., Chieffi, A., \& Limongi, M. 2008, ApJ, 689,1031

Zhang, H. W., Gehren, T., Butler, K., Shi, J. R., \& Zhao, G. 2006, A\&A, 457, 645

Zhao, L., Fischer, D. A., Brewer, J., Giguere, M., \& Rojas-Ayala, B. 2018, AJ, 155,24

\section{Appendix A: Radiogenic heat output of the Earth}

Table A.1. Estimates of radiogenic heat output in the Earth upon its formation and at the present day.

\begin{tabular}{|c|c|c|c|c|c|}
\hline \multirow[b]{2}{*}{ Nuclide $(\mathrm{X})$} & \multicolumn{2}{|c|}{ Concentration (ppb) } & \multirow[b]{2}{*}{$\mathrm{X} / \mathrm{Mg}^{(d)}$} & \multicolumn{2}{|c|}{ Heat $(\mathrm{TW})^{(a)}$} \\
\hline & Present-day ${ }^{(b)}$ & Upon formation $^{(c)}$ & & Present-day & Upon formation \\
\hline${ }^{232} \mathrm{Th}$ & $74.6 \pm 6.8$ & $93.5 \pm 8.5$ & $4.19 \pm 0.38 \times 10^{-7}$ & $7.9 \pm 0.7$ & $9.9 \pm 0.9$ \\
\hline${ }^{235} \mathrm{U}$ & $0.143 \pm 0.014$ & $12.7 \pm 1.3$ & $5.70 \pm 0.58 \times 10^{-8}$ & $0.33 \pm 0.03$ & $29.1 \pm 2.9$ \\
\hline${ }^{238} \mathrm{U}$ & $19.7 \pm 2.0$ & $39.9 \pm 4.0$ & $1.79 \pm 0.18 \times 10^{-7}$ & $7.5 \pm 0.8$ & $15.3 \pm 1.5$ \\
\hline${ }^{40} \mathrm{~K}$ & $28.2 \pm 3.0$ & $353.6 \pm 37.3$ & $1.59 \pm 0.17 \times 10^{-6}$ & $3.2 \pm 0.3$ & $40.6 \pm 4.3$ \\
\hline $\mathrm{Th}+\mathrm{U}+\mathrm{K}$ & $122.6 \pm 7.6$ & $499.6 \pm 38.5$ & $\ldots$ & $19.0 \pm 1.1$ & $94.9 \pm 5.5$ \\
\hline
\end{tabular}

Notes. ${ }^{(a)}$ Calculated by multiplying the corresponding concentrations (upon planet formation and at the present day) with the heat generation rates, $h$, of individual radionuclides (see Table 3). ${ }^{(b)}$ We refer to the mantle concentrations of Wang et al. (2018) (Col. 3 of their Table 1), based on which the radioactive elemental concentrations are converted into the radionuclide concentrations by adopting ${ }^{232} \mathrm{Th} / \mathrm{Th}=1,{ }^{235} \mathrm{U} / \mathrm{U}=0.0072$, ${ }^{238} \mathrm{U} / \mathrm{U}=0.9928$, and ${ }^{40} \mathrm{~K} / \mathrm{K}=1.19 \times 10^{-4}$ (Turcotte \& Schubert 2002) for the present-day bulk silicate Earth. ${ }^{(c)} C_{0}=C_{t} \times \mathrm{e}^{t \ln 2 / T_{1 / 2}}$, where $C_{0}$ and $C_{t}$ represent the abundances of a radionuclide upon the Earth formation $(t=4.56 \mathrm{Ga})$ and at the present day, respectively; $T_{1 / 2}$ is given in Table 3 . ${ }^{(d)} \mathrm{X} / \mathrm{Mg}=C_{0}(\mathrm{X}) / C_{0}(\mathrm{Mg})$, which refers to the early stage of Hadean Earth (i.e. upon the planet formation); $C_{0}(\mathrm{Mg})=C(\mathrm{Mg})=22.3 \pm 0.2 \%(\mathrm{Wang}$ et al. 2018), i.e. the concentration of $\mathrm{Mg}$ is kept unchanged over gelogical time. 